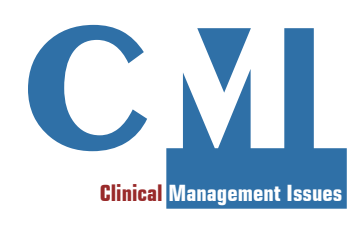

\title{
Basilar Artery Occlusion: Clinical Management and Therapy
}

\begin{abstract}
Basilar artery occlusion is a potentially life-threatening subset of the larger category of posterior circulation strokes, carrying $>80 \%$ fatality rate without treatment. This condition accounts for about $1 \%$ to $4 \%$ of all ischemic strokes and is generally related to local atherothrombosis or cardioembolism.

Diagnosis can be challenging because presenting symptoms are often non focal, such as headache, dizziness, and vertigo. The onset of the symptomatology can be abrupt without preceding events, abrupt with prodromal symptoms, or progressive and stuttering. Finally, the severity of clinical presentation may range from isolated cranial nerve palsies to tetraplegia, locked-in state, or coma. If basilar artery occlusion is readily recognized and confirmed with the aid of neuroimaging, intravenous thrombolysis or endovascular treatment can be undertaken immediately in order to recanalize the occluded artery and thus reduce mortality and improve outcome.
\end{abstract}

Keywords: Stroke; Basilar Artery Occlusion; Headache Disorders; Magnetic Resonance Image; Thrombolysis

Occlusione dell'arteria basilare: gestione clinica e terapia

CMI 2018; 12(1): 67-76

https://doi.org/10.7175/cmi.v12i1.1363
1 Division of Neurology, Department of

Neurosciences, Azienda Ospedaliera "San Pio", Benevento, Italy

\section{INTRODUCTION}

The vertebrobasilar arterial system receives only $20 \%$ of cerebral blood flow and supplies the posterior portion of the brain including the brainstem, the thalami, the cerebellum, and parts of the occipital and temporal lobes $[1,2]$. Posterior circulation ischemia $(\mathrm{PCI})$ is a clinico-pathological condition associated with an infarction within the vertebrobasilar arterial system and is characterized by a complex symptomatology, course, and outcome $[1,2]$.PCI accounts for about $20-25 \%$ of all ischemic strokes and has an adjusted incidence of 18 per 100.000 person-years (95\% confidence interval-CI: 10/100,000 to $26 / 100,000)[1,2]$. Basilar artery occlusion (BAO) is a potentially life-threatening subset of $\mathrm{PCI}$, carrying > $80 \%$ fatality rate without treatment and accounts for about
$1 \%$ to $4 \%$ of all ischemic strokes [3]. BAO still represents one of the most challenging conditions to diagnose and manage. Despite better imaging techniques, in fact, the diagnosis of this condition is often delayed, especially because in many cases presenting symptoms are non-focal, such as headache, dizziness, and vertigo [1-3]. The aim of this article is to provide clinicians with an easy and reliable tool for promptly recognizing and treating $\mathrm{BAO}$.

\section{ETIOLOGY}

The most frequent causes of BAO are local thrombosis and artery-to-artery thromboembolism originating from arteriosclerotic lesions [3]. Other important etiologies are cardiac emboli and vertebral artery dissec-
Corresponding author Marco Sparaco

Division of Neurology, Department of Neurosciences, Azienda Ospedaliera "San Pio", Via Pacevecchia 53, 82100 Benevento, Italy Phone: +0039-0824-57492; Fax: +0039-0824-57293 E-mail: marcosparaco@alice.it

Received: 15 June 2018 Accepted: 20 September 2018 Published: 9 October 2018 
tions, affecting respectively $30-35 \%$ and $6-8 \%$ of patients [3]. Atherosclerosis preferentially involves the proximal and middle segments of the basilar artery (BA), while the occlusion of the distal segment is typically indicative of an arterioembolic or cardioembolic event [3-5].

Vertebrobasilar dolichoectasia, an anatomic variant consisting of enlargement and dilatation of the vertebral/basilar artery, has been shown also to predispose to $\mathrm{BAO}$ through a reduction in flow velocity leading to local thrombus formation $[1,6]$.

Rare causes include arteritis, meningitis, cervical trauma, coagulopathy, aneurysms, hereditary arteriopathies, as well as complications after endovascular procedures and neurosurgery [3].

Finally, we recently described a case of reversible stenosis of the BA due to hemoconcentration, a condition frequently found in psychiatric patients, especially if suffering from a severe depression [7].

\section{CLINICAL FEATURES}

Several aspects, described hereafter, contribute to make $\mathrm{BAO}$ one of the most challenging conditions to diagnose and manage:

\section{Presenting symptoms are often non-focal}

Most common premonitory symptoms of $\mathrm{BAO}$ are dizziness and vertigo, followed by headache and neck pain $[3,8,9]$.

Dizziness and vertigo are two terms used interchangeably by patients to indicate nonspecific symptoms accounting for about 4 million emergency department visits annually in the United States [9]. Dizziness is usually described as a feeling of light-headedness or lack of mental clarity [10]. Vertigo more specifically describes a sensation of spinning and usually indicates dysfunction of the peripheral vestibular or central vestibule-cerebellar system [6]. Vertigo and/or dizziness caused by $\mathrm{BAO}$ are usually associated to other brainstem or cerebellar symptoms [6]. However, when vertigo is the only symptom and the neurologic examination is normal is not easy to establish the central or peripheral origin of the symptomatology. In these cases the head-impulse-nystagmus-test of skew (HINTS) can be a useful bedside test to distinguish between vertigo caused by peripheral or central lesions [11].

In patients with PCI headache is a common presenting symptom that seems to be caused by irritation of trigeminovascular afferents located in brainstem [12]. In a study by Ferbert et al. of 85 patients with $\mathrm{BAO}$, headache was reported in $25.8 \%$ of cases on admission [8].The headache occurred during the 2 weeks before the stroke and had a predominantly occipital localization. The onset was both sudden and the main complaint, so that initially it was considered subarachnoid hemorrhage [8].

\section{The onset of symptomatology is quite variable}

Based on the symptoms onset, three major clinical types of $\mathrm{BAO}$ presentation have been described $[3,13]$ :

- Abrupt without preceding events. This form is characterized by sudden onset of severe motor and bulbar symptoms (i.e. quadriplegia, ophthalmoplegia, and anarthria) combined with reduced consciousness.

\section{- Abrupt with prodromal symptoms.} Transient prodromal symptoms, such as double vision, dysarthria, vertigo, paresthesiae, etc., precede the monophasic BAO symptoms by several days or even months.

- Progressive and stuttering. This form is characterized by gradual or stuttering course of posterior circulation symptoms such as blurred vision, balance disturbance, dysarthria, bilateral paresthesiae, or motor weakness, which finally are associated with a reduced consciousness $[3,13]$.

\section{The clinical picture varies depending on the site and type of the BA obstruction}

Owing to different degrees of the brainstem involvement, the severity of the clinical presentation may range from isolated cranial nerve palsies to tetraplegia, locked-in state, or coma [3].

- Occlusions of the proximal or middle segments of the BA usually cause large pontine strokes with quadriplegia, reduced consciousness, dysarthria and dysphagia, horizontal gaze paresis, and other cranial nerve palsies [3].

- Occlusions in the distal segment of the BA result in strokes bilaterally in the mesencephalon and thalamus with decreased consciousness, quadriparesis, and nuclear or supranuclear oculomotor and pupillomotor dysfunctions [3]. 
- The disorder known as "top of the basilar syndrome" is characterized by visual, oculomotor, and behavioral abnormalities (i.e. somnolence, vivid hallucinations, and dreamlike behavior), often without substantial motor dysfunction $[14,15]$.

- Large pontine strokes resulting from BAO are the most frequent cause of locked-in syndrome, a disorder characterized by quadriplegia, bilateral facial plegia, anarthria, and aphagia [3]. The typical involvement of pontine tegmentum is responsible for horizontal gaze paresis. Patients, that are awake and alert, can communicate just by blinking and vertical eye movements [3].

\section{DIFFERENTIAL DIAGNOSIS}

Any rapidly progressive clinical condition with multiple cranial nerve dysfunctions (i.e. cranial polyradiculitis, Miller-Fisher syndrome, botulism, or myasthenic crisis) can potentially be mistaken for a brainstem lesion.

Toxic or metabolic disturbances, such as drug abuse or hypoglycemia, may initially present with features resembling cerebrovascular disease. Central pontine myelinolysis and Wernicke's encephalopathy usually present with brainstem deficits. A history of rapid correction of hyponatremia or of poor nutritional intake will clarify the diagnosis.

Neuroinflammatory disorders, such as sarcoidosis or Behçet's disease, may acutely affect the brainstem. However, these diseases often have systemic clinical features which are useful for correctly guiding the diagnosis.

Central nervous system (CNS) infection by viruses (i.e. Epstein-Barr virus or West Nile virus), bacteria (i.e., Listeria monocytogenes), or fungi may mimic stroke [16]. Clinical findings, cerebrospinal fluid examination, and magnetic resonance imaging (MRI) features usually help to get a correct diagnosis.

Acute intracranial hemorrhage affecting brainstem, subarachnoid hemorrhage, and tumor mimicking ischemic stroke can be differentiated from BAO only by imaging [16] .

Extensor jerks and spasms and decerebrate posturing arising with $\mathrm{BAO}$ are sometimes mistaken for grand mal seizures [17].

Finally, in the presence of a headache associated with posterior circulation symptoms, a clinician should also consider the diagnosis of a migraine with brainstem aura.
This rare type of primary headache disorder, previously referred to as basilar migraine, is characterized by attacks preceded and/or accompanied by transitory focal neurologic symptoms pointing to dysfunction in the region supplied by the $\mathrm{BA}$ and its branches [18]. The diagnosis is based on the finding of at least two migraine attacks accompanied by at least two of the following fully reversible symptoms: dysarthria, vertigo, tinnitus, impaired hearing, double vision, ataxia, and decreased level of consciousness $[18,19]$.

\section{INVESTIGATION}

Computed tomography (CT), contrastenhanced CT angiography (CTA), MRI, magnetic resonance angiography (MRA), and Doppler sonography can be used in the acute setting to evaluate patients with suspected BAO. If a patient is a candidate for thrombolytic therapy, neuroimaging should be performed as soon as possible to reduce the door to needle time.

- Non-contrast CT (NCCT) is essential to quickly rule out hemorrhagic stroke and other intracranial space occupying lesions. The pc-Alberta stroke program early CT score (pc-ASPECTS) measuring early ischemic changes in 8 different brain regions has been recently proposed as a grading system to evaluate stroke extension and predict outcome in PCI [20]. Unfortunately, in PCI beam hardening due to the skull base, reduces CT sensitivity, and stroke could be identified in the acute phase only in around $20-40 \%$ of patients [21]. A dense basilar artery sign is visible in only about two-thirds of patients, but, when present, it is highly specific for BAO [3]. Contrast-enhanced CT angiography (CTA) has great value to detect vascular occlusions and is increasingly used in comprehensive stroke centers to select candidates for endovascular treatment. Currently, great attention is given to the prognostic value of collateral blood flow in the hyperacute phase of stroke. Recently, two semiquantitative CTA-based grading systems have been proposed to quantify the potential collateral flow in patients with acute BAO: the posterior circulation collateral score (PC-CS) and the basilar artery on Computed Tomography angiography (BATMAN) score $[22,23]$. Both of these 
scores proved to be strong predictors of functional outcome as well as mortality in BAO and can help to select patients for endovascular treatment $[22,23]$. CT whole brain volume perfusion (PCT) measures changes in Hounsfield unit values per pixel over time after contrast injection, providing information about capillary-level hemodynamics of brain parenchyma. PCT allows to measure parameters such as mean transit time (MTT), time to peak (TTP), cerebral blood flow $(\mathrm{CBF})$, and cerebral blood volume (CBV) [24]. A mismatch between a hypoperfused area (reduced $\mathrm{CBF}$ and increased MTT and TTP) and the ischemic core (reduced CBV) may entail the presence of the so-called ischemic penumbra-hypoperfused still viable parenchyma. Therefore, PCT has been used to select patients with strokes with salvageable tissue, eligible for endovascular treatment also outside common time windows [25-27]. Using multimodal CT, encompassing NCCT, CTA and PCT, vertebrobasilar strokes were diagnosed in the acute phase in up to $76 \%$ of patients. Sensitivity for pons/midbrain lesion was around $60 \%[28,29]$.

- MRI is more sensitive than CT in the assessment of $\mathrm{PC}$ infarcts, particularly in the brainstem, and can detect early evidence of infarction with diffusion-weighted imaging (DWI) sequences [1,2,30]. MRIDWI is considered the gold standard to evaluate infarct core [31]. MRI-perfusion weighted imaging (PWI), likewise PCT, can define the hypoperfused brain area during ischemic stroke. MRI diffusionperfusion mismatch may be helpful to select patients for acute recanalization therapies [31]. MRA allows, even without contrast injection, to identify the type and location of BA occlusion or stenosis.

- Angiography is still the gold standard for collateral evaluation in $\mathrm{BAO}$ and is used in the hyperacute phase only for therapeutic purposes $[1,3,9]$.

- Transcranial echo-color-Doppler can show intracranial vascular occlusions $[2,30]$. However, ultrasound investigation is more operator-dependent and less sensitive in the diagnosis of posterior circulation disease than MRA or CTA, and can be rarely used as the sole or primary investigation for the assessment of PC strokes $[1,30]$.

\section{ACUTE TREATMENT}

Acute $\mathrm{BAO}$ treatment aims to restore the cerebral blood flow in the occluded vessel and salvage brain tissue. It has been shown that $\mathrm{BA}$ recanalization can substantially enhance a patient's chances of survival and of good functional recovery [3]. A metaanalysis of 45 studies $(n=2056)$ on acute $\mathrm{BAO}$, in particular, has shown that recanalization of BA results in a two-fold reduction in mortality and 1.5 -fold reduction in death or dependency [32].

Furthermore, the existence of a relationship between the time from symptom onset to arterial recanalization and clinical outcome has been widely demonstrated. As evidenced in the BASICS registry, a significantly increased risk of poor outcome seems to occur when recanalization therapy is started $>6$ hours after the estimated time of $\mathrm{BAO}$ [33].In this regard, it is necessary to clarify the concept of "time to recanalization therapy", i.e. the interval between estimated time of BAO and start of recanalization therapy. Since BAO is preceded by prodromal symptoms in $>60 \%$ of cases $[3,34,35]$, most of these patients are likely to be excluded from any acute therapeutic option if it is considered as an inclusion criterion the time of onset of any symptom to treatment. Therefore, as suggested in the BASICS registry, the estimated time of $\mathrm{BAO}$ is defined as "time of onset of acute symptoms leading to clinical diagnosis of $\mathrm{BAO}$ or, if not known, last time the patient was seen normal before onset of these symptoms" [33].

Acute treatment options for $\mathrm{BAO}$ include intravenous fibrinolysis (IVT), intra-arterial thrombolysis (IAT), and/or endovascular mechanical treatment [1-3,23].

- IVT. It is the first therapeutic strategy introduced for the management of acute ischemic stroke. This treatment was first evaluated in several randomized controlled trials (RCTs) with recombinant tissue plasminogen activator ( $\mathrm{rt}-\mathrm{PA}$ ), such as NINDS, ECASS, ECASS II, and ATLANTIS [36-39]. In particular, the NINDS study found a benefit of IVT when treatment was started within three hours of the onset of symptoms and demonstrated that patients treated with rt-PA were at least $30 \%$ more likely to have a good functional outcome than patients treated with placebo [36]. The safety and efficacy of IVT were then evaluated in three multicenter studies, that are the 
SITS-MOST, the ECAS III, and the IST-3, with a therapeutic window of 3 , 4.5 , and 6 hours, respectively [40-42]. However, the vast majority of data from these studies concern patients with anterior circulation infarcts (ACI). Indeed, data regarding PC strokes in the SITSMOST and ECASS III studies are lacking and only $8.1 \%$ of patients included in the IST-3 trial had PCI [42]. In the last years, several trials performed with angiographic assessment have reported a progressive improvement of the recanalization rate and functional outcome in patients with BAO treated with IV rtPA. In a Finnish single-center consecutive IVT series the rate of recanalization was $65 \%$ and 30 of 116 patients (26\%) had good outcome (modified Rankin Scale [mRS] 0-2) by 3 months [43]. A study by Strbian et al. on 184 patients with angiography-proven $\mathrm{BAO}$ treated with IVT showed that recanalization of $\mathrm{BAO}$ produced good outcomes in $50 \%$ of patients without extensive baseline ischemia (pc-ASPECT $\geq 8)$ [44].

- IAT. Findings from uncontrolled and controlled studies with IAT have shown a high recanalization rate, ranging on average from $63 \%$ to $65 \%[13,45]$. The results of an analysis of 420 non-randomized patients with BAO treated with IAT (82\%) or with IVT (18\%) showed not significant differences between the two groups of $\mathrm{pa}^{-}$ tients in death, dependency, and favorable outcomes, although the recanalization rate was higher in patients treated with IAT (65\% vs. 53\%) [13]. The Basilar Artery International Cooperation Study (BASICS), a prospective, observational study of $\mathrm{pa}^{-}$ tients with $\mathrm{BAO}$, compared efficacy of antithrombotic treatment, IVT and IAT [46]. In patients with mild-to-moderate basilar stroke, a good outcome (mRS 0-2) was achieved in higher proportion in the IV thrombolysis group compared to intra-arterial treatment group (respectively $53 \%$ vs. $30 \%$ ). Outcome did not differ significantly when considering patients with severe stroke ( $\mathrm{mRS} \leq 2$ in $20 \%$ vs. $11 \%)$. Recanalization occurred in $72 \%$ of patients treated with endovascular procedures compared to $63 \%$ of patients treated with IV thrombolysis and was associated with increased independency [46].

- Endovascular mechanical treatment. In the last years, mechanical thrombectomy (MT) has been performed using many different types of devices. Currently, the so-called "stent retrievers" are the most commonly used ones. These new devices can be deployed within the clot, quickly removing the clot and restoring the blood flow once retrieved. Compared to previous treatments, MT methods reach higher recanalization rates and have lower risk of hemorrhages and wider time window $[45,47]$. Recently, an observational, multicenter study, including 148 consecutive patients with $\mathrm{BAO}$ treated with endovascular procedures, the ENDOSTROKE study, reported a good outcome (mRS $0-2$ ) in up to $34 \%$ of treated patients, irrespectively of stroke severity, and complete recanalization in $79 \%$ of cases [48]. More recently, Gory and colleagues analyzed 22 consecutive patients with basilar stroke treated at their hospital with Solitaire ${ }^{\mathrm{TM}}$ stent-retrievers and performed a systematic review of published data (15 studies involving a total of 312 subjects). The recanalization rate reached $81 \%$ (95\% CI: $73 \%$ to $87 \%$ ), good outcome was found in $42 \%$ (95\% CI: $36 \%$ to $48 \%$ ), and symptomatic intracranial hemorrhage was observed in $4 \%$ (95\% CI: $2 \%$ to $8 \%$ ) of cases [49].

Over the past decade, treatment for acute ischemic stroke has evolved to include both IVT and endovascular procedures. Phan et al. analyzed six randomized controlled trials involving 1943 patients to assess the functional outcomes and complications of IVT with and without endovascular treatment. In this study, patients who received IVT with endovascular treatment had significantly higher rates of excellent functional outcomes (mRS 0-1) (95\% CI: 1.29-2.39; RR: 1.75) in comparison with those who received IVT alone. Endovascular treatment increased the RR of a good functional outcome by at least $30 \%$ compared to IVT alone [50].

Perfect timing for endovascular treatment in BAO is not well established. Recently, published American guidelines suggest to perform MT in PCI only within 6 hours from stroke onset [51]. However, most centers, considering the poor prognosis associated with BAO, apply recanalization therapies for this pathology up to $12-24$ hours after symptom onset [52].

An analysis of 19 published cohorts of patients with $\mathrm{BAO}$ showed that higher recanalization rates, such as those obtained with endovascular mechanical treatments, decrease mortality but do not necessarily trans- 
late into better outcomes $[3,53]$. Therefore, even if it is widely agreed that significant survival after $\mathrm{BAO}$ requires rapid access to revascularization, straightforward evidence about efficacy of endovascular therapy (i.e. IAT or MT) over systemic fibrinolysis in basilar stroke is still missing [53].

\section{IN-HOSPITAL MANAGEMENT AND SECONDARY PREVENTION}

Patients with BAO should be carefully monitored with MRI to evaluate burden lesion and cerebellar edema. The latter generally peaks 3 to 5 days after infarction and may cause compression of the fourth ventricle and/or herniation [9]. After initial stabilization, patients should undergo an extensive systematic etiological work-up including the following assessments:

- Laboratory testing: complete blood count, fasting lipids, C-reactive protein, erythrocyte sedimentation rate, coagulation panel, antiphospholipid and/or anticardiolipin antibodies, lupus anticoagulant, and glycated hemoglobin A1c;

- Genetic analysis focusing on inherited prothrombotic states: factor V Leiden/ G1691A, prothrombin/G20210A, etc.;

- MRA (or CTA) and ultrasound investigation of the head and neck: to evaluate for atherosclerotic disease, dissection, vertebrobasilar dolichoectasia, luminal narrowing, or occlusion;

- Echocardiogram: to evaluate for potential cardiac sources of emboli such as a valvular vegetation, ventricular thrombus, etc.
Patients should be placed on continuous cardiac monitoring to detect cardiac arrhythmias (i.e. atrial fibrillation, etc.) and to check the blood pressure. Patients with BAO may be particularly sensitive to changes in blood pressure [9]. However, so far the balance of risk and benefit in lowering blood pressure in these cases is not yet clear. In a study that analyzed the association between blood pressure and stroke in patients undergoing endarterectomy for symptomatic carotid stenosis it was found higher risk of stroke at lower blood pressure, especially if systolic blood pressure $<140$ $\mathrm{mmHg}$ [54]. There are no similar studies in patients with $\mathrm{BAO}$, but the same possibility of hemodynamic insufficiency at lower blood pressures exists. Therefore, caution is advised in lowering the pressure aggressively in these patients, particularly if they have limited or absent posterior communicating arteries as well as other collateral flow abnormalities [1].

The choice of the appropriate medical therapy for secondary prevention of $\mathrm{BAO}$ should be based on the causative stroke mechanism (i.e. cardioembolic, atherosclerotic, etc.) as well as on the risk stratification for recurrence [1-3,30]. Therefore, possible medical treatments include antiplatelet agents or anticoagulants, as well as lipid-lowering drugs. Recommended treatment is detailed in recent guidelines $[51,55]$. In patients with indications for anticoagulation (i.e. atrial fibrillation), treatment should be started generally within two weeks after an acute ischemic stroke, to avoid the risk of hemorrhagic transformation of the infarct.

Keypoints

- $B A O$ is a potentially life-threatening neurologic emergency that requires high suspicion index

- Patients presenting with sudden onset of headache or vertigo need an accurate neurological and neuroradiological evaluation

- Multimodal CT (if available) or MRI must be promptly used in the acute setting to confirm the diagnosis of $B A O$

- As soon as BAO is confirmed with neuroimaging, intravenous thrombolysis or endovascular treatment can be undertaken

- Shortening the time from the symptoms onset to start of the treatment can give patients better chance to survive and avoid severe complications

- Identification causes of BAO is essential for choosing the most appropriate secondary medical therapy

- Screening of uncommon causes of $B A O$ is mandatory

- $M R I$ is a gold standard for follow up of $B A O$ 
PROPOSED ALGORITHM FOR ACUTE BAO MANAGEMENT

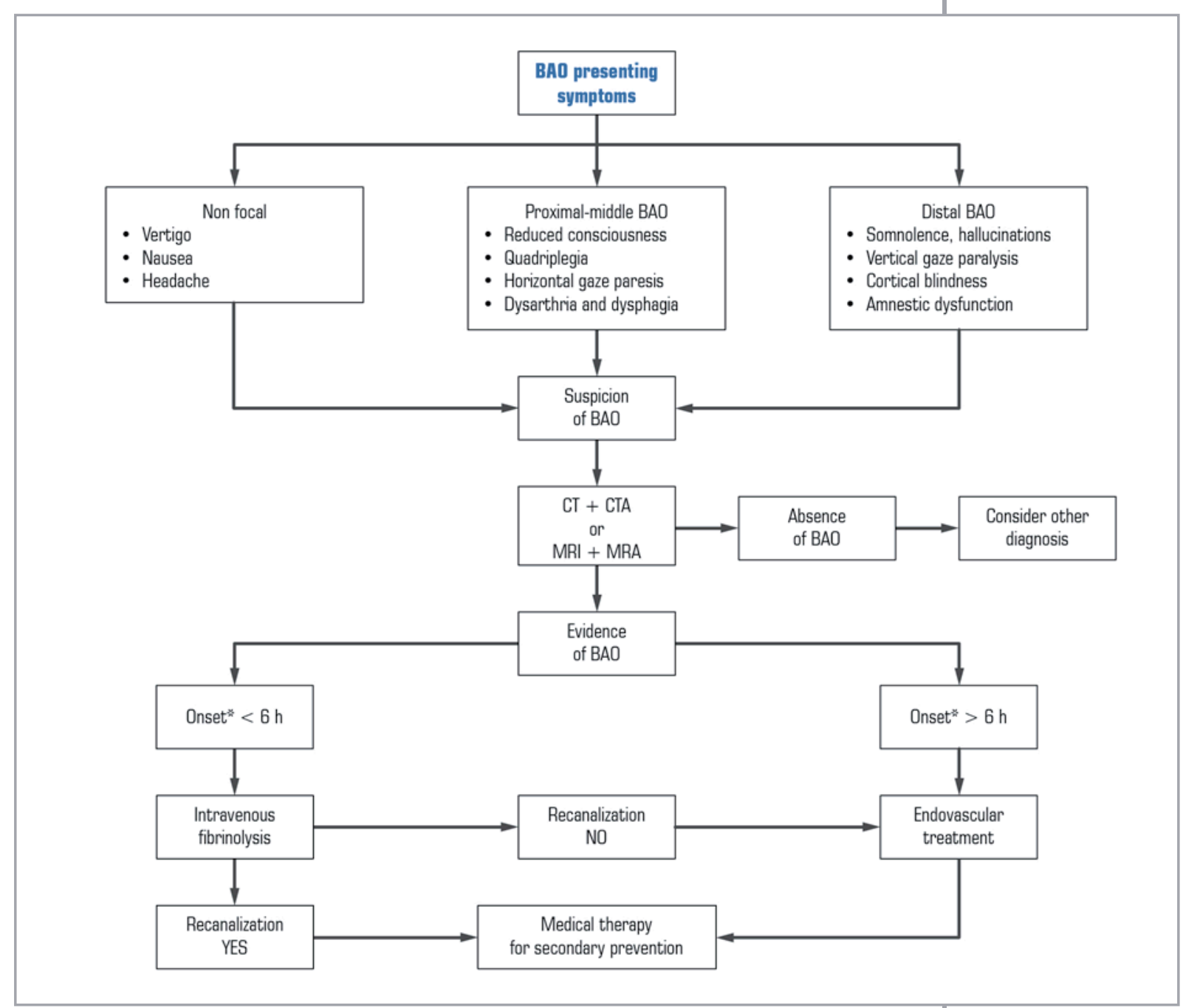

$\mathrm{BAO}=$ basilar artery occlusion; $\mathrm{CT}=$ computed tomography; $\mathrm{CTA}=$ computed tomography angiography; $\mathrm{MRA}=$ magnetic resonance angiography; $\mathrm{MRI}=$ magnetic resonance imaging

*"Time of onset of acute symptoms leading to clinical diagnosis of BAO or, if not known, last time the patient was seen normal before onset of these symptoms" [33].

\section{Funding}

This article has been published without the support of sponsors.

\section{Conflicts of interests}

The author declares he has no competing financial interests concerning the topics of this article.

\section{REFERENCES}

1. Markus HS, van der Worp HB, Rothwell PM. Posterior circulation ischaemic stroke and transient ischaemic attack: diagnosis, investigation, and secondary prevention. Lancet Neurol 2013; 12: 989-98; https://doi.org/10.1016/S1474-4422(13)70211-4

2. Nouh A, Remke J, Ruland S. Ischemic posterior circulation stroke: a review of anatomy, clinical presentations, diagnosis, and current management. Front Neurol 2014; 5: 1-16; https://doi. org/10.3389/fneur.2014.00030

3. Mattle HP, Arnold M, Lindsberg PJ, et al. Basilar artery occlusion. Lancet Neurol 2011; 10 : 1002-14; https://doi.org/10.1016/S1474-4422(11)70229-0

4. Caplan LR, Amarenco P, Rosengart A, et al. Embolism from vertebral artery origin occlusive disease. Neurology 1992; 42: 1505-12 
5. Meyding-Lamadé U, Rieke K, Krieger D, et al. Rare diseases mimicking acute vertebrobasilar artery thrombosis. J Neurol 1995; 242: 335-43

6. Savitz SI, Caplan LR. Vertebrobasilar disease. N Engl J Med 2005; 352: 2618-26; https://doi. org/10.1056/NEJMra041544

7. Sparaco M, Feleppa M. Reversible stenosis of the basilar artery due to transient hemoconcentration: a case report. Neurol Disord Therap 2018; 2; https://doi.org/10.15761/ NDT.1000127

8. Ferbert A, Bruckmann H, Drummen R. Clinical features of proven basilar artery occlusion. Stroke 1990; 21: 1135-42

9. Demel LD, Broderick JP. Basilar artery occlusion: un update. Neurohospitalist 2015; 5: 142-50; https://doi.org/10.1177/1941874415583847

10. Schneider JI, Olshaker JS. Vertigo, vertebrobasilar disease, and posterior circulation ischemic stroke. Emerg Med Clin North Am 2012; 30: 681-93; https://doi.org/10.1016/j.emc.2012.06.004.

11. Kattah JC,Talkad AV,Wang DZ, et al.HINTS to diagnose stroke in the acute vestibular syndrome: three-step bedside oculomotor examination more sensitive than early MRI diffusion-weighted imaging. Stroke 2009; 40: 3504-10; https://doi.org/10.1161/STROKEAHA.109.551234

12. Vestergaard K, Andersen G, Nielsen MI, et al. Headache in stroke. Stroke 1993; 24: 1621-4

13. Lindsberg PJ, Mattle HP. Therapy of basilar artery occlusion: a systematic analysis comparing intra-arterial and intravenous thrombolysis. Stroke 2006; 37: 922-8; https://doi.org/10.1161/01. STR.0000202582.29510.6b

14. Caplan LR. "Top of the basilar" syndrome. Neurology 1980; 30: 72-9

15. Mehler MF. The rostral basilar artery syndrome: diagnosis, etiology, prognosis. Neurology 1989; 39: $9-16$

16. Von Campe G, Regli F, Bogousslavsky J. Heralding manifestations of basilar artery occlusion with lethal or severe stroke $J$ Neurol Neurosurg Psychiatry 2003; 74: 1621-6; https://doi. org/10.1136/jnnp.74.12.1621

17. Ropper AH. 'Convulsions' in basilar artery occlusion. Neurology 1988; 38: 1500-1

18. Kirchmann M, Thomsen LL, Olesen J. Basilar-type migraine: clinical, epidemiologic, and genetic features. Neurology 2006; 66: 880-6; https://doi.org/10.1212/01.wn1.0000203647.48422.dd

19. Headache Classification Committee of the International Headache Society (IHS). The International Classification of Headache Disorders, 3rd edition (beta version). Cephalalgia 2013; 33: 629-808; https://doi.org/10.1177/0333102413485658

20. Puetz V, Sylaja PN, Coutts SB, et al. Extent of hypoattenuation on CT angiography source images predicts functional outcome in patients with basilar artery occlusion. Stroke 2008; 39: 2485-90; https://doi.org/10.1161/STROKEAHA.107.511162

21. Hwang DY, Silva GS, Furie KL, et al. Comparative sensitivity of computed tomography vs. magnetic resonance imaging for detecting acute posterior fossa infarct.J Emerg Med 2012; 42: 559-65; https://doi.org/10.1016/j.jemermed.2011.05.101

22. van der Hoeven EJ, McVerry F, Vos JA, et al. Collateral flow predicts outcome after basilar artery occlusion: the posterior circulation collateral score. Int J Stroke 2016; 11: 768-75; https:// doi.org/10.1177/1747493016641951

23. Alemseged F, Shah DG, Diomedi M, et al. The Basilar Artery on Computed Tomography Angiography Prognostic Score for Basilar Artery Occlusion. Stroke 2017; 48: 631-7; https:// doi.org/10.1161/STROKEAHA.116.015492

24. van Seeters T, Biessels GJ, van der Schaaf IC, et al. Prediction of outcome in patients with suspected acute ischaemic stroke with CT perfusion and CT angiography: the Dutch acute stroke trial (DUST) study protocol. BMC Neurol 2014; 14: 37; https://doi.org/10.1186/14712377-14-37

25. Saver JL, Goyal M, Bonafe A, et al. Stent-Retriever Thrombectomy after Intravenous t-PA vs. t-PA Alone in Stroke. N Engl J Med 2015; 372: 2285-95; https://doi.org/10.1056/ NEJMoa1415061

26. Campbell BCV, Mitchell PJ, Kleinig TJ, et al. Endovascular Therapy for Ischemic Stroke with Perfusion-Imaging Selection. N Engl J Med 2015; 372: 1009-18; https://doi.org/10.1056/ NEJMoa1414792

27. Nogueira RG, Jadhav AP, Haussen DC, et al. Thrombectomy 6 to 24 Hours after Stroke with a Mismatch between Deficit and Infarct. $N$ Engl J Med 2018; 378: 11-21; https://doi. org/10.1056/NEJMoa1706442 
28. van der Hoeven EJ, Dankbaar JW, Algra A, et al. Additional Diagnostic Value of Computed Tomography Perfusion for Detection of Acute Ischemic Stroke in the Posterior Circulation. Stroke 2015; 46: 1113-5; https://doi.org/10.1161/STROKEAHA.115.008718

29. Sporns P, Schmidt R, Minnerup J, et al. Computed tomography perfusion improves diagnostic accuracy in acute posterior circulation stroke. Cerebrovasc Dis 2016; 41: 242-7; https://doi. org/10.1159/000443618

30. Schulz UG, Fischer U. Posterior circulation cerebrovascular syndromes: diagnosis and management. J Neurol Neurosurg Psychiatry 2016; 0: 1-9; https://doi.org/10.1136/jnnp-2015311299

31. Yoo AJ, Hakimelahi R, Rost NS, et al. Diffusion weighted imaging reversibility in the brainstem following successful recanalization of acute basilar artery occlusion. J Neurointerv Surg 2010; 2: 195-7; https://doi.org/10.1136/jnis.2009.002048

32. Kumar G, Shahripour RB, Alexandrov AV. Recanalization of acute basilar artery occlusion improves outcomes: a meta-analysis. JNeurointervent Surg 2014; 0:1-7; https://doi.org/10.1136/ neurintsurg-2014-011418

33. Vergouwen MD, Algra A, Pfefferkorn T, et al. Basilar Artery International Cooperation Study (BASICS) Study Group. Time is brain(stem) in basilar artery occlusion. Stroke 2012; 43: 30036; https://doi.org/10.1161/STROKEAHA.112.666867

34. Ferbert A, Brückmann H, Drummen R. Clinical features of proven basilar artery occlusion. Stroke 1990; 21: 1135-42

35. Baird TA, Muir KW, Bone I. Basilar artery occlusion. Neurocrit Care 2004; 3: 319-30; https:// doi.org/10.1385/NCC:1:3:319

36. The National Institute of Neurological Disorders and Stroke rt-PA Stroke Study Group. Tissue plasminogen activator for acute ischemic stroke. N Engl J Med 1995; 333: 1581-7; https://doi. org/10.1056/NEJM199512143332401

37. Hacke W, Kaste M, Fieschi C, et al.; for the ECASS-study group. Safety and efficacy of i.v. thrombolysis with a recombinant tissue plasminogen activator in the treatment of acute hemispheric stroke. JAMA 1995; 274: 1017-25

38. Hacke W, KasteM, Fieschi C, et al. Randomised double-blind placebocontrolled trial of thrombolytic therapy with i.v. alteplase in acute ischaemic stroke (ECASS II). Second EuropeanAustralasian acute stroke study investigators. Lancet 1998; 352: 1245-51

39. Clark WM, Wissman S, Albers GW, et al. Recombinant tissue-type plasminogen activator (Alteplase) for ischemic stroke 3 to 5 hours after symptom onset. The ATLANTIS Study: a randomized controlled trial. Alteplase thrombolysis for acute non interventional therapy in ischemic stroke. JAMA 1999; 282: 2019-26

40. Wahlgren N, Ahmed N, Dávalos A, et al. SITS-MOST investigators Thrombolysis with alteplase for acute ischaemic stroke in the Safe Implementation of Thrombolysis in StrokeMonitoring Study (SITSMOST): an observational study. Lancet 2007; 369: 275-82; https:// doi.org/10.1016/S0140-6736(07)60149-4

41. Hacke W, Kaste M, Bluhmki E, et al., for the European Cooperative Acute Stroke Study (ECASS) investigators. Alteplase compared with placebo within 3 to 4.5 hours for acute ischemic stroke. NEnglJ Med 2008; 359: 1317-29; https://doi.org/10.1056/NEJMoa0804656

42. The IST-3 collaborative group. The benefits and harms of i.v. thrombolysis with recombinant tissue plasminogen activator within $6 \mathrm{~h}$ of acute ischaemic stroke (the third international stroke trial [IST-3]): a randomised controlled trial. Lancet 2012; 379: 2352-63; https://doi. org/10.1016/S0140-6736(12)60768-5

43. Sairanen T, Strbian D, Soinne L, et al, for the Helsinki Stroke Thrombolysis Registry (HSTR) Group. Intravenous thrombolysis of basilar artery occlusion: predictors of recanalization and outcome. Stroke 2011; 42: 2175-9; https://doi.org/10.1161/STROKEAHA.110.605584

44. Strbian D, Sairanen T, Silvennoinen H, et al. Thrombolysis of basilar artery occlusion: impact of baseline ischemia and time. Ann Neurol 2013; 73: 688-94; https://doi.org/10.1002/ana.23904

45. Rha J-H, Saver JL. The impact of recanalization on ischemic stroke outcome: a meta-analysis. Stroke 2007; 38: 967-73; https://doi.org/10.1161/01.STR.0000258112.14918.24

46. Schonewille WJ, Wijman CAC, Michel P, et al. On behalf of the BASICS study group. Treatment and outcomes of acute basilar artery occlusion in the Basilar Artery International Cooperation Study (BASICS): a prospective registry study. Lancet Neurol 2009; 8: 724-30; https://doi.org/10.1016/S1474-4422(09)70173-5

47. Knap D, Kirmes T, Honkowicz M, et al. Treatment of basilar artery occlusion using various techniques of interventional radiology. Vasc Med Surg 2016; 4: 1; https://doi.org/10.4172/23296925.1000241 
48. Singer OC, Berkefeld J, Nolte $\mathrm{CH}$, et al. Mechanical recanalization in basilar artery occlusion: The ENDOSTROKE study.Ann Neurol 2015; 77: 415-24; https://doi.org/10.1002/ana.24336

49. Gory B, Eldesouky I, Sivan-Hoffmann R, et al. Outcomes of stent retriever thrombectomy in basilar artery occlusion: an observational study and systematic review. J Neurol Neurosurg Psychiatry 2016; 87: 520-5; https://doi.org/10.1136/jnnp-2014-310250

50. Phan K, Zhao DF, Phan S, et al. Endovascular therapy including thrombectomy for acute ischemic stroke: A systematic review and meta-analysis with trial sequential analysis. J Clin Neurosci 2016; 29: 38-45; https://doi.org/10.1016/j.jocn.2015.12.025

51. Powers WJ, Rabinstein AA, Ackerson T, et al. 2018 Guidelines for the Early Management of Patients With Acute Ischemic Stroke: A Guideline for Healthcare Professionals From the American Heart Association/American Stroke Association. Stroke 2018; 49: e46-e99; https:// doi.org/10.1161/STR.0000000000000158

52. Lindsberg PJ, Pekkola J, Strbian D, et al. Time window for recanalization in basilar artery occlusion: Speculative synthesis. Neurology 2015; 85: 1806-15; https://doi.org/10.1212/ WNL.0000000000002129

53. Lindsberg PJ, Sairanen T, Nagel S, et al. Recanalization treatments in basilar artery occlusion-Systematic analysis. European Stroke Journal 2016; 1: 41-50; https://doi. org/10.1177/2396987316629889

54. Rothwell PM, Howard SC, Spence D. Relationship between blood pressure and stroke risk in patients with symptomatic carotid occlusive disease. Stroke 2003; 34: 2583-90; https://doi. org/10.1161/01.STR.0000094424.38761.56

55. The European Stroke Organisation (ESO) Executive Committee and the ESO Writing Committee. Guidelines for Management of Ischaemic Stroke and Transient Ischaemic Attack 2008. Cerebrovasc Dis 2008; 25: 457-507; https://doi.org/10.1159/000131083 